\title{
0-16
}

\section{Blistering and re-deposition on tungsten exposed to ASDEX Upgrade divertor plasma}

\author{
M. Balden*, V. Rohde, S. Lindig, A. Manhard, K. Krieger, and ASDEX Upgrade Team \\ Max-Planck-Institut für Plasmaphysik, EURATOM Assoziation, Boltzmannstr.2, D-85748 Garching.
}

\begin{abstract}
A well-polished and pre-characterised polycrystalline tungsten specimen was exposed to the outer divertor plasma of ASDEX Upgrade close to the strike point for 510 discharges in the campaign 2011 by using the divertor manipulator system. The exposure with a mixture of hydrogen and various impurities leads to blistering. The exposure parameters are comparable to those used in blistering studies under laboratory conditions. Furthermore, spherical dust particles formed by the strong plasma contact were transported and deposited onto the nearly vertically orientated target plates, i.e., the analysed specimen. In subsequent discharges, these particles were partly eroded and a microscopic pattern of layered deposits was formed around them with an elongation rotated $\sim 40^{\circ}$ to the projected B field line direction.
\end{abstract}

PACS: 28.52 Fa, 52.40 Hf, 68.37 Hk, 82.80 Ej

Keywords: Tungsten, Erosion \& Deposition, Surface Analysis, Dust, Blister

Corresponding and presenting author: Martin Balden

Max-Planck-Institut für Plasmaphysik, Boltzmannstr.2, D-85748 Garching

Email: Martin.Balden@ipp.mpg.de 


\section{Introduction}

Morphological changes of the surface of the plasma-facing material by the power and particle loads from a fusion plasma can have major impact on erosion resistance, fuel uptake and thermo-mechanical behaviour of the material surface. Blistering of tungsten (W) materials - caused by the large transient hydrogen supersaturation in the metal - is a phenomenon well documented in laboratory experiments [1-7], but only sparsely observed in fusion devices $[8,9]$. The laboratory experiments indicate that the tungsten grade and the surface quality, e.g., polished or technical surface, as well as the impurity content in the incident hydrogen flux play a role in appearance and abundance of blistering and other morphological changes [10,11,12,13]. E.g., a small fraction of helium in the incident ion flux prevents blistering [12]. In a burning fusion device, there will be always a small fraction of $\mathrm{He}$, but $\mathrm{He}$ is also present in today's devices due to the frequently performed He glow discharge cleaning. Furthermore, seeding gases for power dissipation, such as $\mathrm{N}_{2}$, and eroded material from the plasma-facing components will impact together with the hydrogen and could also affect the occurrence of blisters on tungsten. Therefore, more studies regarding the occurrence of blisters on $\mathrm{W}$ by hydrogen impact with a typical admixture of impurities in the percent range under fusion-relevant exposure conditions in the divertor region close to the strike point should be performed. Well-polished surfaces should be used to allow comparison to results from laboratory studies.

Another aspect of surface morphologies is the effect of roughness on the erosiondeposition pattern on microscopic scale. The impurities, such as $\mathrm{W}, \mathrm{C}$ or Be, in the incident flux of the plasma lead to deposition of material. The effect of net deposition or erosion depends sensitively on the flux ratios [14]. Roughness of the surface can lead to local net deposition even in erosion-dominated areas, e.g., close to the strike point in the outer divertor $[15,16]$. Recently a model was developed that calculates the element-resolved incident flux distribution for a pre-defined surface roughness in order to describe the observed erosiondeposition pattern [17]. This model was benchmarked with surface analysis results on ASDEX Upgrade (AUG) tiles. The observed and modelled microscopic deposition leads, together with erosion, to a levelling of the surface [17]. Due to the importance of predicting 
the wall composition development of ITER and DEMO with operation time and the contribution of deposited layers to the fuel retention $[18,19,20]$, the microscopic development should be studied in detail on thoroughly pre-characterised surfaces.

The aim of this study was to investigate both processes - blistering and microscopic erosion-deposition pattern formation - in a dedicated material exposure experiment in AUG. Well-polished, pre-characterized W specimens with several well-defined surface structures were exposed to the outer divertor plasma in the campaign 2011 and analysed with several microscopic techniques.

\section{Experimental}

Two polycrystalline bulk tungsten specimens were exposed and examined. Prior to the exposure, both $\mathrm{W}$ specimens were polished according to the procedure described in [7] to remove the distorted layer produced by machining. Therefore, a direct comparison with blistering studies in laboratory devices is possible [7,21]. Furthermore, some rectangular trenches serving also as markers were cut with a focused ion beam (FIB) into the polished surface as artificial morphologies with side walls perpendicular to the surface. The polished surface and the trenches were analysed with scanning electron microscopy (SEM). The used microscope (Helios NanoLab600, FEI) allows in situ SEM imaging, FIB cutting, i.e., crosssectioning, and elemental analysis with energy dispersive X-ray spectroscopy (EDX). Note for preparation of cross-sections, the surface was coated artificially by a Pt-C-layer. For obtaining the height of surface features a confocal laser scanning microscope (CLSM) (LEXT OLS4000, Olympus) and an atomic force microscope (AFM) in ac mode (MFP-3D, Asylum Research) were used.

The microscopic analysis focuses on the specimen exposed as an inlay of the AUG divertor manipulator system, which is described in reference [22]. The specimen was exposed in the outer divertor for 510 discharges in the campaign 2011 with 2500 s divertor plasma, leading to a cumulative ion fluence of $\sim 6 \times 10^{25} \mathrm{~m}^{-2}$ (Fig. 1). The strike point was for most of the time a few centimetres below the specimen (position marked in Fig. 1), i.e., the specimen 
was mainly exposed to the scrape-off layer divertor plasma and not to the private flux region plasma. The power loads to the specimen and, therefore, the material temperature varied from discharge to discharge and even in each discharge. The temperature is estimated to range from 400 to $800 \mathrm{~K}$, with some short excursions to even higher temperatures. The ion impact energy is in the order of tens to hundreds of $\mathrm{eV}$. These exposure parameters fall into the parameter range of blistering studies in laboratory experiments [1-13].

The second specimen is a Langmuir probe of the outer divertor [23], which was installed the whole campaign 2011 with 5360 s divertor plasma. The strike-point distribution for the whole campaign 2011, the position of this specimen, and the obtained total fluence to it are given in Fig. 1, too. This specimen was only used to confirm some observations from the other specimen.

\section{Results and discussion}

Fig. 2 (a) shows an area of about $640 \times 640 \mu \mathrm{m}^{2}$ with one marker as well as a large number of features originate from the exposure in AUG. Only the marker was observable on the polished surface before exposure to AUG divertor plasma. Many of the features in Fig. 2 (a) exhibit an aligned elongation. The orientation of the main elongation axis is not parallel to the main plasma impact angle calculated from the magnetic configurations. Instead, this elongation is rotated by about $40^{\circ}$ against the B field line direction projected onto the surface of the nearly vertical target plate (for geometry see [22,23]). Typically, the upper-left end of the elongated features is higher and they tail out to the lower-right end, e.g., Fig. 2(b-d). The lateral dimensions of the highest part are comparable to its height (Fig. 2(c)). Furthermore, the height profiles level out smoothly in all directions (Fig. 2(c,d)).

Beside the elongated features, circular features are also observed. Some of them are very flat (height to diameter ratio of $<0.1$ ) and hard to detect, while a few others have a height comparable to their diameter. By analysing at higher magnifications in SEM, the latter ones are identified as spherical dust particles, and the former ones as blisters. The nature of the blisters was confirmed unambiguously by cross-sectioning several of them using FIB (Fig. 3). 
The observed crack system runs along grain boundaries about parallel to the surface in a depth of the order of a fraction of the blister diameter. This is typical also for blisters observed in laboratory experiments on rolled $\mathrm{W}[7,21]$ and the same formation mechanisms can be assumed. The hydrogen gas filling, as observed in laboratory studies [21], is not confirmed yet. The statistical analysis of a larger area on the specimen leads to sizes in the range of $1-20 \mu \mathrm{m}$, an areal density of the blisters of nearly $10^{9} \mathrm{~m}^{-2}$ and a cap thickness of below $2 \mu \mathrm{m}$. These values are comparable to values observed in laboratory experiments in the studied parameter range $[5,7,21]$. Again as in the laboratory experiment, it is not observed any loss of blister caps.

By cross-sectioning the individual elongated features (Fig. 4), about spherical particles with a composition and inner morphology typical for dust in AUG [24,25] are found or at least there remains. The particles consist either of pure $\mathrm{W}$, dominantly $\mathrm{B}$, or a conglomerate of B, C and W. These particles are partly eroded (left side in Fig. 4(c,d)). In addition, the tail (Fig. 2) is clearly formed from several re-deposited layers with some porosity. The redeposited material consists mainly of tungsten, but contains also some B, N, C, and O. Such composition and structure are typical for re-deposited layers in AUG [16]. The observation of partial erosion and layered re-deposits indicates that the elongated features are representative for a large fraction of discharges in AUG (Fig. 1) and are not merely produced in single unusual discharges.

By counting all elongated features also as dust particles and taking their width perpendicular to the elongation as the diameter of the spherical dust particle, an areal density of around $10^{9} \mathrm{~m}^{-2}$ and a diameter range of $0.5-10 \mu \mathrm{m}$, with a mean value slightly above 1 $\mu \mathrm{m}$, is determined. The spherical shape implies strong plasma contact of the particles leading to melting, evaporation and ablation of material. It could be expected that the particles flow together with the plasma and arriving at the divertor target plate. The formation process and the transport of the dust particles will not be discussed here.

Also on the analysed specimen used as Langmuir probe, the elongated features with incorporated dust particles are observed and their orientation is again $\sim 40^{\circ}$ rotated against the projected B field line direction. Furthermore, on both specimens, re-deposited layers partially 
fill the trenches artificially introduced by FIB (Fig. 2(a)). The outer morphology of the layers in trenches shows some elongated structure with the same orientation as the elongation of the re-deposits around the dust particles. Deposition is even observed on the vertical side wall of the trenches. The thickness and the inner morphology of the layer in the trenches were not yet further evaluated.

From the modelling by K. Schmid et al. [17] it is expected that the impacting ions from the plasma hit the specimen with angles to the surface normal of roughly $20-30^{\circ}$ with a spread of about $\pm 5^{\circ}$ around these values and, therefore, erode elevated regions. The promptly re-deposited material, which leads to the observed layers in the shadowed regions, arrives nearly perpendicular to the surface with a spread of about $\pm 10^{\circ}$ [17]. This spread is, by itself, not sufficient to explain the observed re-deposition of material underneath the overhanging parts of the spheres (Fig. 4 (d)). It is suggested that this part of re-deposition is created by reflection of impacting impurities as well as the sputter deposition of material previously deposited on the dust particles and the surrounding flat substrate. Reflected and sputtered particles would leave the surface with about a cosine distribution and should be able to reach and fill the gap underneath the sphere without ionisation in between. These effects as well as the dynamic evaluation of the surface morphology must be implemented in future modelling. A challenge will also be to explain and confirm the observed rotation of the deposition pattern. Existing ERO modelling does not predict such strong rotation [26,27].

Overall, the observed morphologies are ideal input data for benchmarking models of the microscopic erosion-deposition pattern and such data should be collected even from different locations in the AUG and other devices. Such models are necessary to predict the evolution of the compositional change of the wall and, therefore, their overall erosion behaviour in future fusion devices.

\section{Summary}

The exposure of polished polycrystalline tungsten specimens to the outer AUG divertor plasma close to the strike point leads to two different morphology changes: Blistering and 
deposition of dust particles with subsequent formation of deposition patterns around them.

The exposure to plasmas containing wall material species and residual impurities of varying concentration (He, N, W, C, B, and O) in the divertor leads to formation of blisters with sizes, areal density and inner structures comparable to those found in laboratory experiments on polished $\mathrm{W}$ surfaces under similar exposure conditions $[7,22]$. Therefore, the same formation mechanisms can be assumed.

On the nearly vertical target plate surfaces, such as that of the analysed specimen, spherical dust particles of varying composition arrive with a rate of several $10^{5}$ particles per $\mathrm{m}^{2}$ and $\mathrm{s}$. The observed compositions - pure W, B-dominated and W-B-C conglomerates - are typical for dust in AUG [24,25]. By far the most observed particles are spherical due to their previous immersion in the plasma. After their arrival on the $\mathrm{W}$ specimen surface, they become partly eroded in subsequent discharges and an acclivity of re-deposited material is formed around them. This material exhibits a layered structure with some porosity and is oriented in the direction opposite to the eroded side. The elongation is rotated about $40^{\circ}$ against the $\mathrm{B}$ field line direction projected onto the specimen surface. The deposited material contains $\mathrm{W}, \mathrm{B}, \mathrm{C}, \mathrm{O}$, and $\mathrm{N}$, as it was observed earlier in such layered re-deposits in AUG [16].

Due to the flat initial surface and the well-defined spherical shape of the surface morphology, the erosion and deposition around a single dust particle is ideal input data for benchmarking models.

\section{References}

[1] A.A. Haasz et al., J. Nucl. Mater. 266-269 (1999) 520.

[2] W. Wang et al., J. Nucl. Mater. 299 (2001) 124-131.

[3] K. Tokunaga et al., J. Nucl. Mater. 307-311 (2002) 126-9.

[4] V.Kh. Alimov et al., J. Nucl. Mater. 337-339 (2005) 619.

[5] M. Fukumoto et al., J. Nucl. Mater. 375 (2008) 224-8. 
[6] S. Lindig et al., Phys. Scripta T138 (2009) 014040.

[7] A. Manhard, Ph.D. Thesis, TU Munich, 2012 (Technical Report IPP 17/34, Garching, 2012).

[8] M. Tokitani et al., J. Nucl. Mater. 337-339 (2005) 937-941.

[9] M. Tokitani et al., J. Nucl. Mater. 390-391 (2009) 156-159.

[10] D. Nishijima et al., Nucl. Fusion 45 (2005) 669.

[11] Y. Ueda et al., Nucl. Fusion 44 (2004) 62.

[12] V.Kh. Alimov et al., Phys. Scripta T138 (2009) 014048.

[13] O.V. Ogorodnikova et al., Phys. Scripta T145 (2009) 014034.

[14] D. Naujoks, W. Eckstein, J. Nucl. Mater. 220-222 (1995) 993-996.

[15] M. Mayer et al., J. Nucl. Mater. 363-365 (2007) 101-106.

[16] A. Kallenbach et al., J. Nucl. Mater. 415 (2011) S19-S26.

[17] K. Schmid et al., Nucl. Fusion 50 (2010) 105004.

[18] K. Schmid et al., J. Nucl. Mater. 415 (2011) S284-8.

[19] M. Reinelt et al., J. Nucl. Mater. 415 (2011) S305-9.

[20] R. Doerner, J. Nucl. Mater. 363-5 (2007) 32-40.

[21] M. Balden et al., J. Nucl. Mater. 414 (2011) 69-72.

[22] K. Krieger et al., J. Nucl. Mater. 241-243 (1997) 684-9.

[23] K. Sugiyama et al., Nucl. Fusion 50 (2010) 035001.

[24] V. Rohde et al., Phys. Scripta T138 (2009) 014024.

[25] N. Endstrasser et al., Phys. Scripta T145 (2011) 014021.

[26] A. Kirchner, Nucl. Fusion 40 (2000) 989.

[27] L. Aho-Mantila et al., J. Nucl. Mater. 415 (2011) S231. 


\section{Figures captions:}

Fig. 1 (single column): Fluence obtained from Langmuir probes (solid symbols) and strike point distribution obtained from magnetic reconstruction (open symbols) on the outer divertor target tile versus the poloidal position in S-coordinate for the 510 discharges of the exposure on the divertor manipulator (stars) and for the whole campaign 2011 of AUG (triangles). The poloidal dimension of the inlay specimen of the divertor manipulator is marked by the two vertical solid lines, while the dashed vertical line indicates the centre of the analysed Langmuir probe (S3ua6). A schematic drawing of the AUG divertor geometry with the definition of the S-coordinate is given, e.g., in reference [23].

Fig. 2 (two columns): (a) Top-view CLSM intensity image of a large area showing one artificial marker (top left) and many elongated features. (b) CLSM image in higher magnification of the green framed central area of (a) containing blisters and elongated features (dust particles with an acclivity of re-deposited material). The numbered lines through one of the elongated features indicate the position of the height profiles given in (c). The profiles are vertically shifted by 0.5 and $1 \mu \mathrm{m}$ for better visibility. The same feature is visible in the centre of the 3D image (d) with the viewing direction from the bottom of (a). The height axis of (d) is 4 times stretched compared to the lateral dimensions. The red arrows in (a) and (b) indicate the B field line direction projected onto the surface. Note for (b) and (d) differential interference contrast was used which accentuate the roughness on flat surface (for details see [7]).

Fig. 3 (two columns): (a) Top-view SEM micrograph. The dashed and dotted line indicates the position of the cross-sections prepared by FIB shown in (b) and (c), respectively. The surface, white area in (b,c), which is tilted by $52^{\circ}$ to the viewing plane, is covered for the FIB cutting with a Pt-C coating. The cross-sectioned area in $(b, c)$ is tilted by 
$38^{\circ}$ to the viewing plane. The black arrow in (a) indicates the B field line direction projected onto the surface.

Fig. 4 (two columns): Top-view SEM micrograph with two features, a spherical dust particle and a typical elongated feature with its head containing a dust particle and a tail of redeposited material. Both are cross-sectioned at the position indicated by the lines. The respective cross-section images are given for the dotted line in (b) and the dashed line in (c). The elemental composition of the spherical particles is determined by EDX. (d) is another example of dust particle with its tail of re-deposited material cross-sectioned along its elongation. The cross-sectioned area in (b-d) is tilted by $38^{\circ}$ to the viewing plane. The black arrow in (a) indicates the B field line direction projected onto the surface. 
Figure 1:

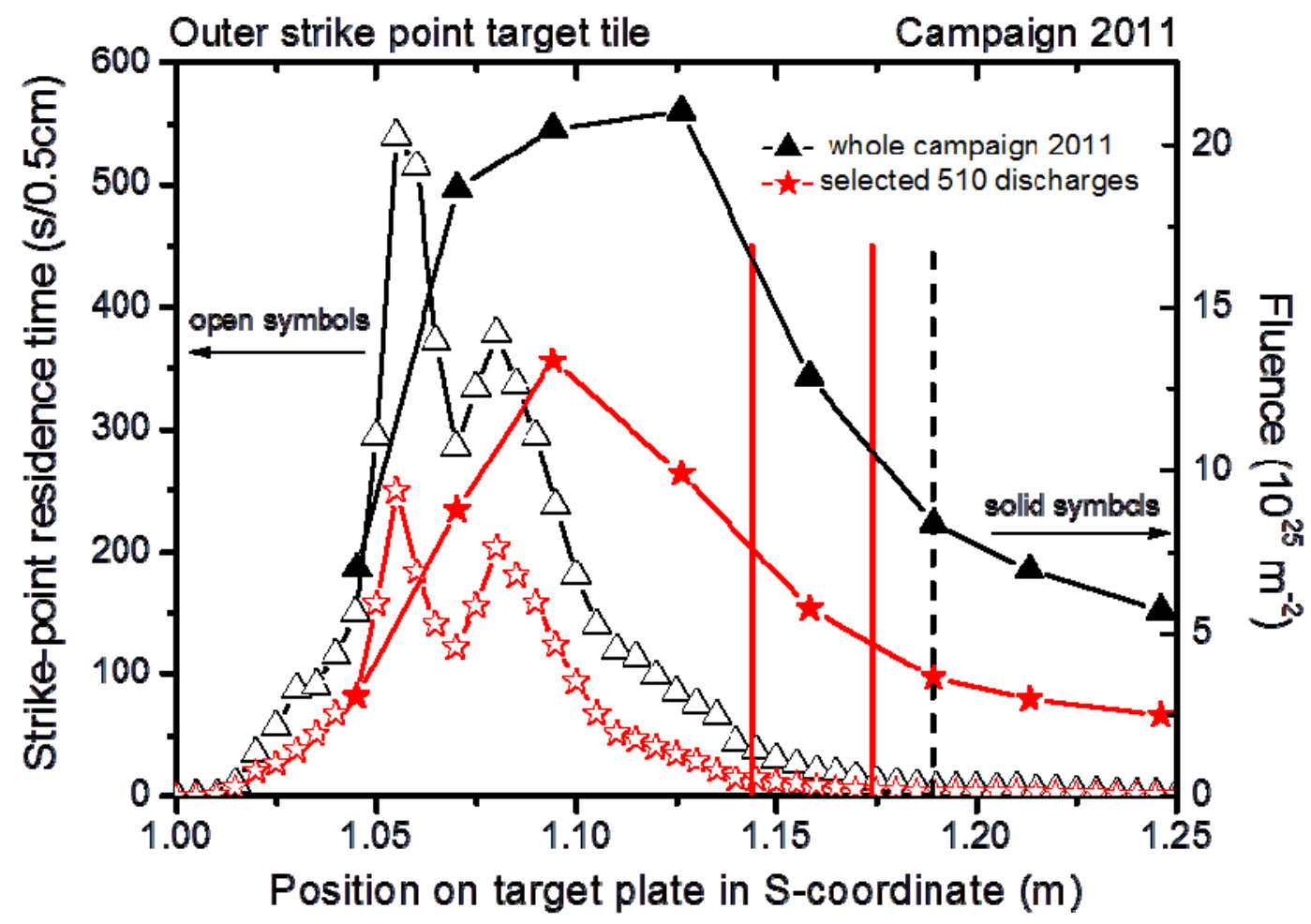


Figure 2:
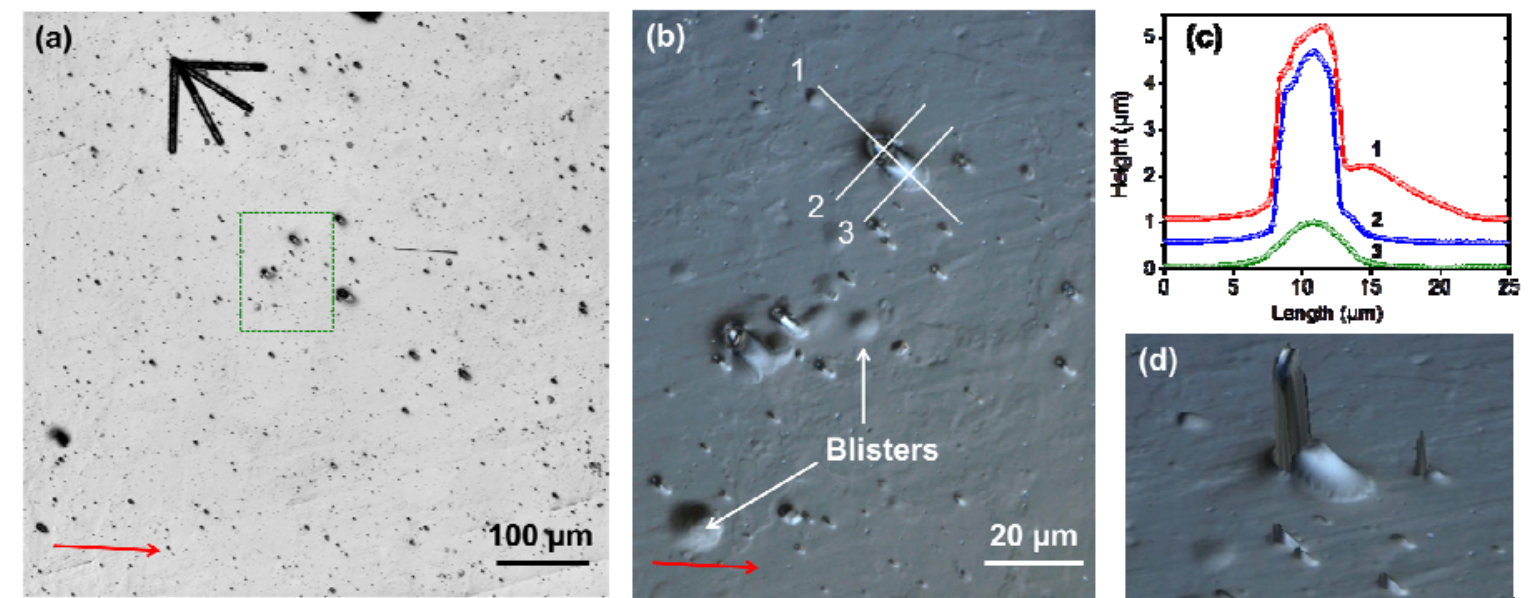
Figure 3:
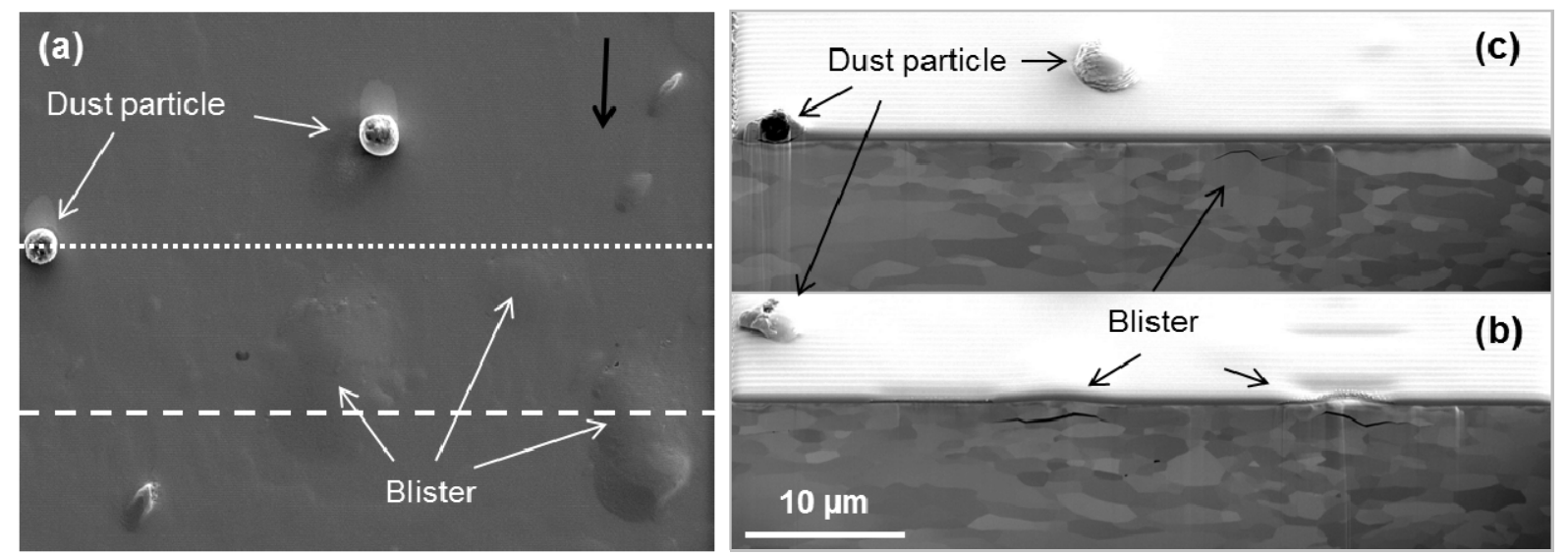
Figure 4:
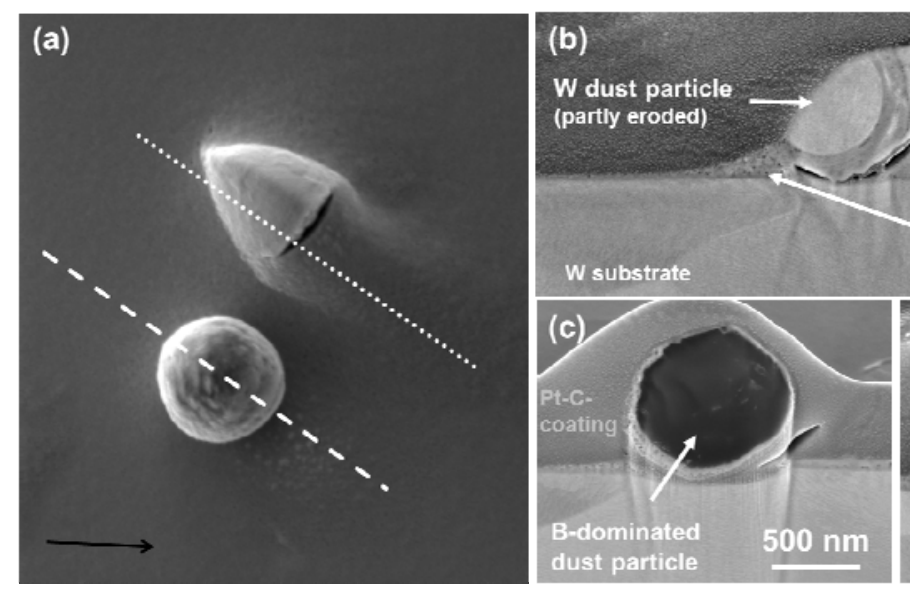

Artificial

t-C-coating

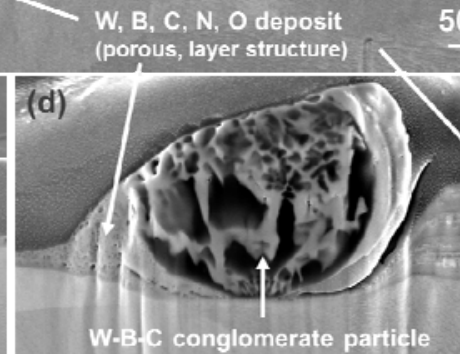

$500 \mathrm{~nm}$

Artificial Pt-C-coating

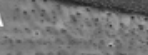

